\title{
Concurrent Reactivation of Varicella Zoster Virus and Herpes Simplex Virus in an Immunocompetent Child
}

\begin{abstract}
Latency within the nervous system is a characteristic feature of herpesviridae infection. It is reactivated by triggering factors such as UV exposure, stress, and trauma. Simultaneous reactivation of herpes simplex and herpes zoster is uncommon, however, an observation provably explained by differences in the trigerring mechanism. Concurrent reactivation of herpes simplex virus (HSV) and varicella zoster virus (VZV) is occasionally encountered in immunosuppressed patients; on the other hand, it is rarely reported in immunocompetent individuals. We present the case of an immunocompetent 8-yr-old female patient with concurrent reactivation of HSV on the face and VZV on the right L2 dermatome.
\end{abstract}

Key Words : Herpesvirus 3, Human; Simplexvirus; Herpesvirus 1, Human

\author{
Hyun-Ho Park, Mu-Hyoung Lee \\ Department of Dermatology, College of Medicine, \\ Kyunghee University, Seoul, Korea \\ Received : 4 July 2003 \\ Accepted : 8 September 2003 \\ Address for correspondence \\ Mu-Hyoung Lee, M.D. \\ Department of Dermatology, College of Medicine \\ Kyunghee University, 1 Hoegi-dong, \\ Dongdaemoon-gu, Seoul 130-702, Korea \\ Tel : +82.2-958-8501, Fax : +82.2-969-6538 \\ E-mail : mhlee@khmc.or.kr
}

\section{INTRODUCTION}

Neurotrophism and latent infection are common characteristics of herpes virus (1-3). Concurrent reactivation of herpes simplex virus (HSV) and varicella zoster virus (VZV), however, is rare, and this may be due to their different biologic features such as recurrence rate, age of reactivation, reactivating cause, resident site in latency, etc. Until now, the simultaneous reactivation of the two herpesviruses has mainly been reported in immunocompromised patients (4-10), probably because immunosuppressed patients are predisposed to infection and because the clinical features in immunosuppresed patients are more severe and extensive.

We report here an immunocompetent patient with concurrent reactivation of $\mathrm{HSV}$ and VZV.

\section{CASE REPORT}

An 8-yr-old female presented with asymptomatic grouped vesicles on the right lower eyelid and right upper gluteal region and thigh, which appeared simultaneously the day before visit. She had no history of chronic diseases other than recurrent herpes simplex on the right lower eyelid over the previous 2 yr. On physical examination, no abnormal findings other than the skin lesions on the right lower eyelid and the right L2 dermatome were observed (Fig. 1). Chest radiography and laboratory tests, including complete blood cell count, liver function test, level of BUN and $\mathrm{Cr}$, level of immunoglobulins, T cell subset counts, and HIV antibody were in normal ranges. Serologic tests were positive for VZV and HSV IgG, weakly positive for VZV IgM, although they were negative for HSV IgM. For further testing, PCR for HSV and VZV DNA were performed using skin obtained from the lesions on the face and thigh. Results were positive for HSV and negative for VZV for the facial skin and vice versa for the thigh skin (Fig. 2). She was subsequently diagnosed with herpes simplex of the face and herpes zoster of the right L2 dermatome and was treated with famciclovir at a dose of $125 \mathrm{mg}$ three times a day for 7 days; both groups of lesions resolved following the treatment.

\section{DISCUSSION}

HSV and VZV are members of the herpesvirinae subfamily. These viruses characteristically cause recurrent infection following latency within the dorsal root and trigeminal ganglia and possess the ability to shift between the latent site in the sensory ganglia and the corresponding innervated skin (1-3). Despite these similarities, their biological properties differ significantly and such differences may explain why concurrent reactivation is rare.

Among other things, herpes zoster and herpes simplex differ in the frequency of recurrence and age at reactivation (1). HSV is reactivated several times during life, but VZV reactivation generally occurs only once through life. VZV reactivation is associated with aging, with the highest incidence rate occurring after $60 \mathrm{yr}$ of age, probably due to a decline in cellular immune response with age (11). HSV is more fre- 
quently reactivated in children and the recurrence declines with age due to maturation of immune response (12). The reactivating cause also differs between these viruses. HSV is reactivated by predisposing factors such as UV exposure, trauma, fever, and stress, but the provocation factors are less apparent in the case of VZV (8). This may be due to direct influence this factors on HSV gene expression (2). The sites of viral dormancy are also thought to be different; HSV lies latent in neuronal cells and VZV in satellite cells $(1,2)$. Consequently, reactivated HSV causes skin lesions limited to the immediate area of innervation of the infected neurons, while reactivated VZV brings about extensive viral proliferation and cell-to-cell infection in the larger dermatomal area $(1,2)$. VZV occasionally cause extensive inflammation, atrophy, and scarring of the dorsal horn and root ganglion, which results in postherpetic neuralgia (13).

The infrequency of reports on simultaneous recurrence of
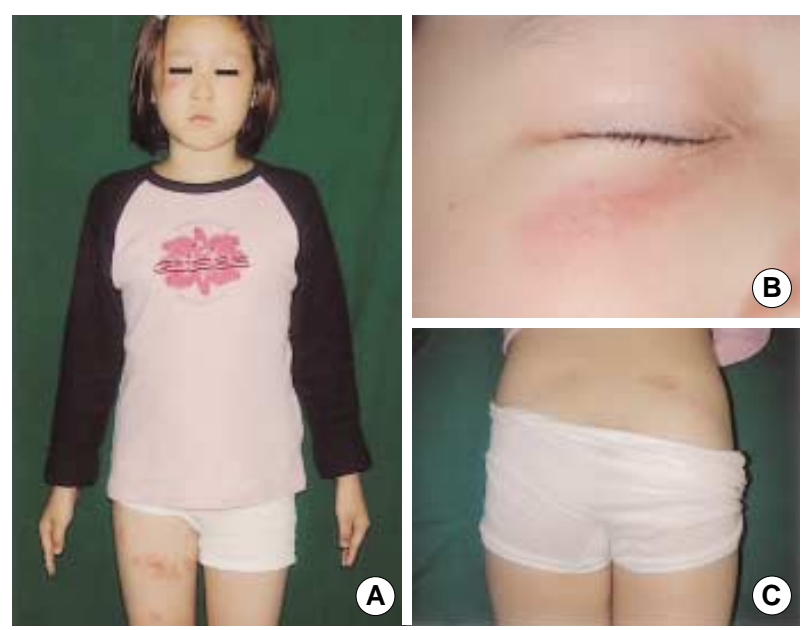

Fig. 1. (A) Grouped vesicles on the right cheek and right $L 2$ dermatome. (B) Grouped multiple vesicles on the infraorbital area. Herpes simplex virus was detected in this lesion. (C) Grouped vesicles and erythematous patches over the L2 dermatome. Varicella zoster virus was detected in these lesion. herpes simplex and herpes zoster may be explained by the differences in the rate of viral reactivation, frequency of viral reactivation, factors that trigger viral reactivation, and histologic sites of viral latency (10). However, it is difficult to determine whether the course of disease are altered by each virus because there is only small number of reports of simultaneous herpes simplex and herpes zoster (8).

Report on concurrent reactivation of HSV and VZV are rare. We found seven reports on concurrent infection of them in the literature (4-10). All patients in the reports were adolescent or adult and their age distribution is from 22 to 84 yr old. Five patients among them were in the immune-suppressed condition or chronic illness such as kidney recipient (4), AIDS (5), lung cancer (6), tuberculosis (7), idiopathic thrombocytopenic purpura (8) and two others are healthy patients $(9,10)$. Gibney et al. (4) suggested that reactivation of a latent virus is associated with depression of cell-mediated immune responses due to primary infection with another herpesvirus. Vivo et al. (6) reported VZV infection on the right V2 dermatome and HSV infection on the right side of palate in a 84-yr-old male with non-small-cell lung cancer; they suggested that a common triggering event may exist to reactivate both viruses in the same sensory ganglion.

These viruses do infect healthy children and adults but the manifestations are more severe and extensive in the immunocompromised individuals. Although some of the reactivated patients were healthy, most of the patients had chronic or immunocompromising diseases. These reports show that concurrent reactivation of HSV and VZV is generally preceded by immunologic abnormalities.

The subject of this study is a healthy child with no history of medical problems and no abnormality in laboratory data, including immunologic parameters. Serologic tests have only small role in the diagnosis of recurrent herpes simplex and late stage of herpes zoster because of seroconversion. However, positive VZV IgG and weakly positive VZV IgM suggest acute VZV reactivation on the thigh. Positive HSV IgG and negative HSV IgM suggest recurrent herpes simplex on

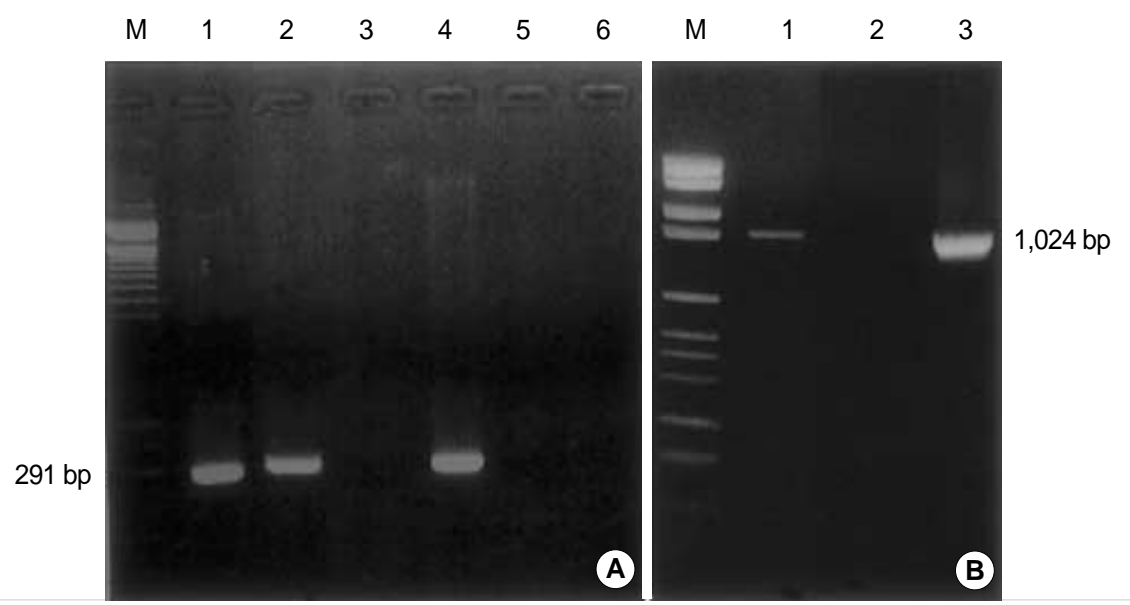

Fig. 2. (A) HSV PCR electrophoresis. Lane M, 100 bp DNA size marker; Lane 1, Positive control; Lane 2, HSV detected in patient's face; Lane 3, HSV is not detected in patient's thigh; Lane 4, Positive control; Lane 5, Negative control (PCR); Lane 6, Negative control (prep). (B) VZV PCR electrophoresis. Lane M Marker VI; Lane 1, VZV detected in the patient's thigh; Lane 2, VZV is not detected in the patient's face; Lane 3, Positive control. 
the face. PCR supported the differential diagnosis of HSV and VZV on the other sites.

Concurrent reactivation of HSV and VZV is generally occurred in the immunocompromised patients and rarely reported in the immunocompetent patients. To date no report has been made on concurrent reactivation of these viruses in children; this is probably due to a low incidence of pediatric VZV infection compared with that in adults (14).

The present case is unique in that the patient is an immunocompetent child. The authors believe this to be the first reported case of simultaneous reactivation of HSV and VZV in different skin regions in an immunocompetent child. However, our case suggest that HSV and VZV could occasionally overlapped regardless of general condition and age. And it is suggested that the reactivation of HSV would have depressed cell mediated immunity and allowing reactivation of VZV, or vice versa.

\section{REFERENCES}

1. Meier JL, Straus SE. Comparative biology of latent varicella zoster virus and herpes simplex virus infections. J Infect Dis 1992; 166 : S13-23.

2. Strauss SE. Clinical and biological differences between recurrent herpes simplex virus and varicella-zoster virus infections. JAMA 1989; 262: 3455-8.

3. Liesegang TJ. Biology and molecular aspects of herpes simplex and varicella-zoster virus infections. Ophthalmology 1992; 99: 781-99.
4. Gibney MD, Leonardi CL, Glaser DA. Concurrent herpes simplex and varicella zoster infection in an immunocompromised patient. $J$ Am Acad Dermatol 1995; 33: 126-9.

5. Cupps TR, Straus SE, Waldmann TA. Successful treatment with acyclovir of an immunodeficient patient infected simultaneously with multiple herpesviruses. Am J Med 1981; 70: 882-6.

6. Vivo CD, Bansal MG, Olarte M, Grossman ME. Concurrent herpes simplex type 1 and varicella-zoster in the V2 dermatome in an immunocompetent patient. Cutis 2001; 68: 120-2.

7. Kahn G. Zoster and herpes simplex. Arch Derm 1967; 95: 298.

8. Nikkels AF, Frere P, Rakic L, Fassotte M, Evrard B, De Mol P, Pierard GE. Simultaneous reactivation of herpes simplex virus and varicella-zoster virus in a patient with idiopathic thrombocytopenic purpura. Dermatology 1999; 199: 361-4.

9. Kandil E. Herpes simplex in the course of zoster. Acta Derm Venereol 1969; 49: 436-7.

10. Binet O, Petit G, Rabary G, Vilmer C, Ferchal F. Simultaneous infection with two viruses from the herpesviridae family: herpes simplex virus and varicella-zoster virus. Press Med 1983; 32: 1998.

11. Miller AE. Selective decline in cellular immune response to varicella-zoster in the elderly. Neurology 1980; 30: 582-7.

12. Berger R, Florent G, Just M. Decrease of the lymphoproliferative response to varicella-zoster virus antigen in the aged. Infect Immun 1981; 32: 24-7.

13. Cunningham AL, Dworkin RH. The management of post-herpetic neuralgia. BMJ 2000; 321: 778-9.

14. Papadopoulos AJ, Birnkrant AP, Schwartz RA, Janniger CK. Childhood herpes zoster. Cutis 2001; 68: 21-3. 